OPEN ACCESS

Edited by:

Matteo Bologna,

Sapienza University of Rome, Italy

Reviewed by:

Weise David,

Universitätsklinikum Leipzig, Germany

Antonella Macerollo,

University College London,

United Kingdom

*Correspondence:

Daniel Zeller

zeller_d@ukw.de

tThese authors have contributed equally to this work

Specialty section:

This article was submitted to

Movement Disorders,

a section of the journa

Frontiers in Neurology

Received: 28 October 2018

Accepted: 22 February 2019

Published: 15 March 2019

Citation:

Odorfer TM, Homola GA, Reich MM,

Volkmann J and Zeller D (2019)

Increased Finger-Tapping Related

Cerebellar Activation in Cervical

Dystonia, Enhanced by Transcranial

Stimulation: An Indicator of

Compensation?

Front. Neurol. 10:231

doi: 10.3389/fneur.2019.00231

\section{Increased Finger-Tapping Related Cerebellar Activation in Cervical Dystonia, Enhanced by Transcranial Stimulation: An Indicator of Compensation?}

\author{
Thorsten M. Odorfer ${ }^{1 \dagger}$, György A. Homola ${ }^{2 \dagger}$, Martin M. Reich ${ }^{1}$, Jens Volkmann ${ }^{1}$ and \\ Daniel Zeller ${ }^{1 *}$ \\ ${ }^{1}$ Department of Neurology, University of Würzburg, Würzburg, Germany, ${ }^{2}$ Department of Neuroradiology, University of \\ Würzburg, Würzburg, Germany
}

Background: Cervical dystonia is a movement disorder causing abnormal postures and movements of the head. While the exact pathophysiology of cervical dystonia has not yet been fully elucidated, a growing body of evidence points to the cerebellum as an important node.

Methods: Here, we examined the impact of cerebellar interference by transcranial magnetic stimulation on finger-tapping related brain activation and neurophysiological measures of cortical excitability and inhibition in cervical dystonia and controls. Bilateral continuous theta-burst stimulation was used to modulate cerebellar cortical excitability in 16 patients and matched healthy controls. In a functional magnetic resonance imaging arm, data were acquired during simple finger tapping before and after cerebellar stimulation. In a neurophysiological arm, assessment comprised motor-evoked potentials amplitude and cortical silent period duration. Theta-burst stimulation over the dorsal premotor cortex and sham stimulation (neurophysiological arm only) served as control conditions.

Results: At baseline, finger tapping was associated with increased activation in the ipsilateral cerebellum in patients compared to controls. Following cerebellar theta-burst stimulation, this pattern was even more pronounced, along with an additional movement-related activation in the contralateral somatosensory region and angular gyrus. Baseline motor-evoked potential amplitudes were higher and cortical silent period duration shorter in patients compared to controls. After cerebellar theta-burst stimulation, cortical silent period duration increased significantly in dystonia patients.

Conclusion: We conclude that in cervical dystonia, finger movements-though clinically non-dystonic - are associated with increased activation of the lateral cerebellum, possibly pointing to general motor disorganization, which remains subclinical in most body regions. Enhancement of this activation together with an increase of silent period 
duration by cerebellar continuous theta-burst stimulation may indicate predominant disinhibitory effects on Purkinje cells, eventually resulting in an inhibition of cerebello-thalamocortical circuits.

Keywords: cervical dystonia, functional MRI, cortical excitability, transcranial magnetic simulation (TMS), continuous theta burst stimulation (cTBS), motor-evoked potentials (MEP), cortical silent period

\section{BACKGROUND}

Cervical dystonia (CD) is a movement disorder leading to involuntary muscle contractions which cause repetitive and twisting head movements and abnormal, sometimes painful head postures (1). Dystonic disorders have been regarded as psychogenic diseases for decades (2) before pathophysiological research provided evidence for underlying basal ganglia dysfunction $(3,4)$. Only over the last years, a growing body of evidence points to the cerebellum (CRB) as an important node in dystonia pathophysiology (5-8). Most of this evidence originates from magnetic resonance imaging (MRI) studies, particularly from advanced techniques like functional $(9,10)$ and resting-state MRI (11), voxel-based morphometry (VBM) (12-15), or probabilistic tractography (16) in different cohorts of dystonia patients. While this leaves little doubt as to cerebellar involvement in dystonic disorders, the exact nature of this involvement remains unclear. In the case of cervical dystonia, functional imaging faces additional challenges: While brain activation associated with dystonic head movement would be of particular interest, data acquisition requires subjects to keep the head still. As the interpretation of task-free functional imaging studies in CD may be ambiguous $(17,18)$, simple hand motor tasks have been used instead to study activation patterns in functional MRI (fMRI). Although clinically non-dystonic, such hand movements have been shown to be associated with altered activity in ipsilateral putamen, insula and cingulate cortex (19) as well as caudate nucleus, putamen and thalamus (20). In an upper limb force task, increased severity of CD was associated with decreased functional activity of the somatosensory cortex and increased activity of CRB (21). Only recently, Prudente et al. used a new paradigm to assess the functional imaging correlate of isometric head movements (22). They found increased activation of the anterior $\mathrm{CRB}$ during constant tension on muscles rotating the head into the pathological direction of torticollis (22). However, while fMRI is able to reveal brain activity associated with a certain condition, the technique is unable to discriminate pathophysiological from compensatory activation. Here, additional neurophysiological approaches like repetitive transcranial magnetic simulation (rTMS) can prove

Abbreviations: AMT, active motor threshold; BOLD, blood oxygen level dependent; CD, cervical dystonia; CGI-I, Clinical Global Impression Improvement subscale; CRB, cerebellum; CSP, cortical silent period; CTRL, control group; cTBS, continuous theta-burst stimulation; EMG, electromyography; FDI, first dorsal interosseous muscle; fMRI, functional magnetic resonance imaging; iTBS, intermittent theta-burst stimulation; M1, primary motor cortex; MEP, motorevoked potential; PD, Parkinson's disease; PMd, dorsal premotor cortex; RMT, resting motor threshold; rTMS, repetitive transcranial magnetic stimulation; SD, standard deviation; TWSTRS, Toronto Western Spasmodic Torticollis Rating Scale. useful (23): By interfering with neuronal processes of a specific brain area, the functional role of this region can be probed (24). In this way, for instance, rTMS over the premotor cortex has been shown to improve symptom severity in CD patients (25). Moreover, a significant reduction of the Toronto Western Spasmodic Torticollis Rating Scale (TWSTRS) score following a 2 week cerebellar continuous theta-burst stimulation (cTBS) treatment has been reported (26). However, similar clinical improvement of $\mathrm{CD}$ has recently been described in a study applying cerebellar intermittent TBS (iTBS) over 10 working days (27), along with an improved performance in the pegboard task, i.e., an enhancement of motor function in a non-dystonic body part (27). From a mechanistic point of view, the results of these two studies appear conflicting: Given their opposite impact on excitability at the primary motor cortex (28), one might not expect that both stimulation protocols can induce clinical improvement when applied to the cerebellum. However, as cerebellar physiology and cytoarchitecture is largely different from the motor cortex, effects of cTBS on M1 may not easily be transferred one-to-one to the CRB. Therefore, rather than a dichotomic issue, the behavioral impact of cerebellar TBS might be considered a net effect of various neuromodulative effects of different direction.

In the present study, we applied a complementary approach to challenge the role of CRB in CD: First, we examined functional MRI (fMRI) brain activation during a simple finger tapping task along with neurophysiological measures of cortical excitability in CD patients as compared to healthy controls. Second, we assessed the effects of an excitability-modulating TMS protocol at the lateral CRB on (i) finger-tapping associated brain activation in fMRI, (ii) measures of cortical excitability, and (iii) clinical scores of CD severity. Changes in physiological and/or clinical measures were anticipated to allow an informed interpretation of fMRI data later-on.

\section{METHODS}

\section{Participants}

Sixteen patients (7 females) with idiopathic cervical dystonia (CD) were recruited from our outpatient clinic for movement disorders. Neurological or psychiatric conditions other than $\mathrm{CD}$ led to exclusion from the study. All CD patients were treated with botulinum neurotoxin injections on a regular basis. The experiments were scheduled at an interval of at least 10 weeks from the last injection, with no or minor treatment effects remaining as judged both by the experimenter and by the patient. In addition, a control group (CTRL) of 16 healthy volunteers matched for age and sex (6 females) was recruited. Handedness was determined by a modified version 
of the Edinburgh Handedness Inventory (29). The protocol conformed to the principles of the declaration of Helsinki and was approved by the Ethics Committee of the Medical Faculty at the University of Würzburg. All participants gave their written informed consent for participation in the study.

\section{Study Design}

Participants were randomized to two arms of the study (fMRI or TMS), with eight CD patients and eight controls per arm (Figure 1). In the TMS arm, participants underwent an electrophysiological work-up before and after cTBS at dorsal premotor cortex (PMd) and CRB, respectively, or sham stimulation (three sessions). In the fMRI arm, brain activation during simple finger tapping was assessed before and after cTBS at PMd or CRB (two sessions). In support of feasibility, the fMRI arm did not comprise an additional sham condition to reduce the single patient's burden within the study. The reason for using two experimental groups, rather than doing all experiments in one group, was the long total duration of five sessions which may overtax the compliance of participants [see also (30)].

\section{TMS and EMG Recording}

All participants received high resolution MRI including T1weighted (T1w) 3D MP-RAGE sequences (1 mm isotropic) to allow the localization of cortical regions by neuro-navigation (Brainsight, Rogue Research, Montreal, Canada). TMS was applied by a MC-B70 double coil connected to a MagPro X100 stimulator (Medtronic A/S 2140 Skovlunde, Denmark).

Electromyography (EMG) was recorded from first dorsal interosseous muscle (FDI) via surface cup electrodes with the reference placed over the metacarpophalangeal joint of the index finger. Signals were amplified using a differential amplifier (CED 1902, Cambridge Electronic Design, Cambridge, UK) and bandpass-filtered between 1 and $200 \mathrm{~Hz}$. EMG signals were sampled at $5,000 \mathrm{~Hz}$ and digitized by an analog-converter (CED 1401 plus, Cambridge Electronic Design, Cambridge, UK).

The left motor hotspot (M1), defined as the optimal position for eliciting motor-evoked potentials (MEPs) in the right FDI muscle, was localized both functionally (TMS) and according to the landmarks described previously (31), with excellent congruence of the two. PMd was considered to be represented in the posterior part of the middle frontal gyrus, which was located around $2 \mathrm{~cm}$ anterior and $1 \mathrm{~cm}$ medial to the motor hot spot (32, 33). $\mathrm{CRB}$ was marked $3 \mathrm{~cm}$ lateral and $1 \mathrm{~cm}$ inferior to the inion (31, 34-36). Targeting M1 and PMd, the coil was held in a $45^{\circ}$ angle to the sagittal plane with the handle in backward direction, while during cerebellar stimulation, the handle pointed upwards.

Resting motor threshold (RMT) was defined as the lowest stimulation intensity evoking MEP amplitudes of at least 50 $\mu \mathrm{V}$ in 5 out of 10 trials (monophasic pulse-shape). Active motor threshold (AMT) was determined during voluntary FDI activation at about 20\% of maximal innervation (visual feedback) and defined as the lowest stimulation intensity evoking MEP amplitudes of at least $200 \mu \mathrm{V}$ in 5 out of 10 attempts (biphasic pulse-shape).

\section{Continuous Theta-Burst Stimulation (cTBS)} cTBS was applied at 80\% AMT (biphasic pulse shape) for a total duration of $40 \mathrm{~s}$ (total amount of 600 pulses) (28). Cerebellar stimulation was applied bilaterally (left side first, $60 \mathrm{~s}$ break between stimulations), while unilateral stimulation of the left PMd and unilateral cerebellar SHAM stimulation (20\% AMT, outer edge of the TMS coil touching the back of the head) served as control conditions.

\section{fMRI Arm}

fMRI (Magnetom Trio, Siemens, Munich, Germany) data [EPI, $3 \mathrm{~mm}$ isotropic, repetition time (TR) $=3,000 \mathrm{~ms}$, echo time (TE) $=30 \mathrm{~ms}$, 164 volumes] were acquired during a straightforward tapping task of the right index finger and thumb before and after cTBS. Via a simple block design paradigm (plus and minus signs) visually presented with OLED goggles [NordicNeuroLab AS (NNL), Bergen, Norway] patients were instructed to press buttons on a response grip (NNL) in a moderate frequency or to rest for the same duration of $30 \mathrm{~s}$. The two conditions were run equally in a randomized order over a total time frame of $8 \mathrm{~min}$. Feedback data was recorded with high accuracy (Presentation, Neurobehavioral Systems Inc., Berkeley, CA, USA.) To minimize artifacts due to head movements, the participant's head was properly fixed during image acquisition.

\section{TMS Arm}

The MEP amplitude (mean of 30) at 130\% RMT was taken as an estimate of corticospinal excitability. The CSP duration (mean of 10) as recorded during voluntary FDI pre-innervation (about $20 \%$ of maximal innervation) at 150\% AMT (biphasic stimulation) was taken as a measure of cortical inhibitory mechanisms. Neurophysiological measures were recorded in the same sequence (RMT-MEP-AMT-CSP) before and after cTBS intervention.

\section{Clinical Assessment}

Clinical severity of $\mathrm{CD}$ was rated on the motor subscale of the TWSTRS (37) and the TSUI scale (38) in a blinded manner by providing standardized video sequences of $\mathrm{CD}$ examination to an experienced clinical investigator uninvolved in the experiment. In addition, $\mathrm{CD}$ patients were asked to rate their personal impression of symptom improvement or deterioration after cTBS by using the Clinical Global Impression Improvement subscale CGI-I (39).

\section{Data Analysis}

First level and group analysis of the fMRI data was carried out with FEAT, part of the FMRIB Software Library (FSL v5.0, FMRIB, Oxford, UK) (40, 41) (FMRIB Software Library). Fieldmap-based distortion-correction was applied to unwarp the data to increase registration accuracy. MCFLIRT was applied for motion estimation and correction (40). Finger tapping feedback data was added as an additional event variable to account for motor activation and variance. A $2 \times 2 \times 2$ design was set up to test for site and group differences and also to verify that there has been no significant baseline variance between runs on different days. A whole brain correlation analysis was performed with 


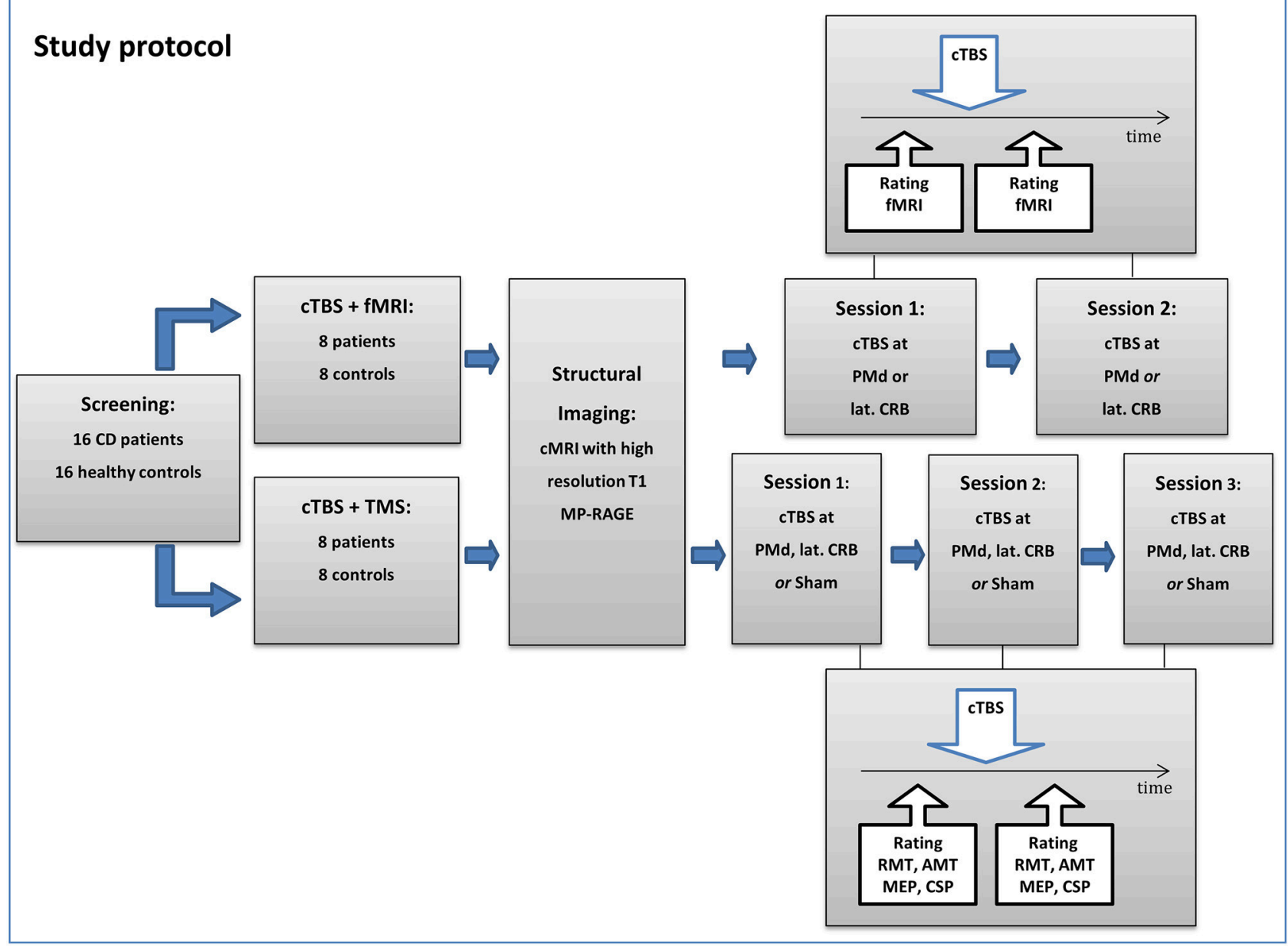

FIGURE 1 | Flowchart of the study protocol. CD, cervical dystonia; cTBS, continuous theta-burst stimulation; TMS, transcranial magnetic stimulation; fMRI, functional magnetic resonance imaging; PMd, dorsal premotor cortex; lat. CRB, lateral cerebellum; RMT, resting motor threshold; AMT, active motor threshold; MEP, motor-evoked potential; CSP, cortical silent period.

cluster thresholding to correct for multiple comparisons. This method is based on Gaussian random field theory and is more sensitive to activation than a voxel based thresholding and is also less overly-conservative with respect to the familywise error rate than the Bonferroni correction (42).

GraphPad Prism (GraphPad Software, San Diego, CA, USA) was used for statistical analyses of TMS data. MEP amplitudes were measured peak-to-peak and averaged. CSP duration was determined by the time interval from MEP onset to the restarting point of EMG activity with $50 \%$ amplitude of pre-MEP level. We tested for normality using the Anderson-Darling-Test. In case of normal distribution, baseline TMS data were compared by two-tailed $t$-tests, otherwise by non-parametric Mann-Whitney$\mathrm{U}$ test. Repeated measures two-way ANOVA was applied to compare between the three stimulation conditions within each group, and Sidak's multiple comparisons test was used for posthoc analysis. Effects were considered significant if $p<0.05$. If not stated otherwise, all values are given as mean \pm standard deviation (SD).

\section{RESULTS}

Demographics and clinical baseline data of $\mathrm{CD}$ patients are shown in Table 1.

\section{fMRI}

At baseline, finger tapping of the right hand was associated with brain activation in the right cerebellar hemisphere and left motor cortex region across groups. Activation of the right lateral CRB was significantly increased in CD patients as compared to healthy controls (Figure 2A, MNI152 coordinates X 21, $\mathrm{Y}-54, \mathrm{Z}-18$ ). Following bilateral cerebellar cTBS, this increased activation was even more pronounced in CD patients (Figure 2B, MNI152 coordinates X 19, Y-59, Z-16). Two other significantly increased activations were found adjacent to the gyrus angularis (MNI152 X-57, Y-42, Z 21) and adjacent to the postcentral sulcus (MNI152 X-55, Y-27, Z 48, Figure 2C). Comparison within the patient group (cTBS on CRB vs. baseline or vs. cTBS on PMd) also showed these elevated activations at the 
TABLE 1 | Clinical characteristics of patients.

\begin{tabular}{lccllcc}
\hline Patient no. & Age* & $\begin{array}{l}\text { Age of } \\
\text { onset* }\end{array}$ & $\begin{array}{l}\text { Dominant } \\
\text { pattern }\end{array}$ & $\begin{array}{l}\text { Second } \\
\text { pattern }\end{array}$ & $\begin{array}{c}\text { TWSTR } \\
\text { baseline }\end{array}$ & $\begin{array}{c}\text { TSUI- } \\
\text { score } \\
\text { baseline }\end{array}$ \\
\hline 1 & & & & & & \\
2 & $41-45$ & $31-35$ & LC right & TC left & 14 & 3 \\
3 & $45-50$ & $41-45$ & TC left & LS right & 15 & 5 \\
4 & $51-55$ & $11-15$ & DHT & LC left & 15 & 6 \\
5 & $55-60$ & $31-35$ & TC left & SE left & 19 & 5 \\
6 & $61-65$ & $16-20$ & DHT & TC right & 22 & 13 \\
7 & $41-45$ & $35-40$ & DHT & TC left & 16 & 4 \\
8 & $41-45$ & $41-45$ & TC left & LC left & 21 & 6 \\
9 & $45-50$ & $21-25$ & TC left & DHT & 21 & 9 \\
10 & $61-65$ & $56-60$ & TC left & LC right & 19 & 3 \\
11 & $36-40$ & $26-30$ & RC & DHT & 17 & 4 \\
12 & $51-55$ & $35-40$ & DHT & TC left & 16 & 8 \\
13 & $61-65$ & $55-60$ & TC left & LC right & 20 & 6 \\
14 & $51-55$ & $51-55$ & TC right & DHT & 24 & 13 \\
15 & $46-50$ & $41-45$ & TC left & DHT & 21 & 6 \\
16 & $51-55$ & $51-55$ & LC right & SE right & 22 & 10 \\
\hline Means & $46-50$ & $41-45$ & DHT & LC left & 18 & 5 \\
\hline SD & $51.9^{* *}$ & 38.5 & & & 18.8 & 6.6 \\
& 7.5 & 13.5 & & & 3.0 & 3.2 \\
\hline & & & & & & \\
\hline
\end{tabular}

*presented in age of range in order to avoid providing indirectly identifiable patient data. ${ }^{* \star}$ for comparison, healthy control group: mean age $45.0 \pm 15.6$ years $(p=0.125)$. TWSTRS, Toronto Western Spasmodic Torticollis Rating Scale; LC, laterocollis; TC, torticollis; LS, lateral shift; $D H T$, dystonic head tremor; SE, shoulder elevation; RC, retrocollis; $A C$, anterocollis.

same locations and at the same significance levels with only minimal differences. In contrast, bilateral cerebellar cTBS had no significant effect on brain activation in healthy controls, and PMd stimulation had no effect on tapping-related fMRI activation in both groups. Continuous monitoring of motor performance (timing, duration and frequency) did not reveal significant correlation between groups or stimulation sites.

\section{TMS}

At baseline, MEP amplitudes were significantly higher $(2.6 \pm$ 1.4 vs. $1.3 \pm 1.0 \mathrm{mV}, p=0.002)$ and CSP duration significantly lower $(132 \pm 23$ vs. $147 \pm 26 \mathrm{~ms}, p=0.036)$ in $\mathrm{CD}$ patients as compared to controls (Figure $\mathbf{3 A}$ ). RMT was lower in CD patients (54.5 \pm 16.7 vs. $65.5 \pm 12.1, p=0.019)$, while AMT was comparable between groups $(p=0.216)$. In CD patients, repeated measures two-way ANOVA with the factors STIMULATION MODE [PMd, CRB, sham] and TIME [pre, post] revealed a significant effect of the factor TIME $\left[F_{(1,21)}=19.59, p=\right.$ 0.0002] on CSP duration. Post-hoc analysis showed a significant increase of CSP duration following CRB stimulation (123 \pm $27 \mathrm{~ms}$ vs. $130 \pm 29 \mathrm{~ms}$; adjusted $p=0.004$ ), but not after PMd or sham stimulation (Figure 3B). In controls, repeated measures two-way ANOVA with the same factors revealed a significant effect of the factor TIME $\left[F_{(1,21)}=5.565, p=0.028\right]$ and a significant interaction effect of STIMULATION MODE $\mathrm{x}$ TIME $\left[F_{(2,21)}=3.636, p=0.044\right]$ on CSP duration. Post-hoc analysis showed a significant increase of CSP duration following PMd
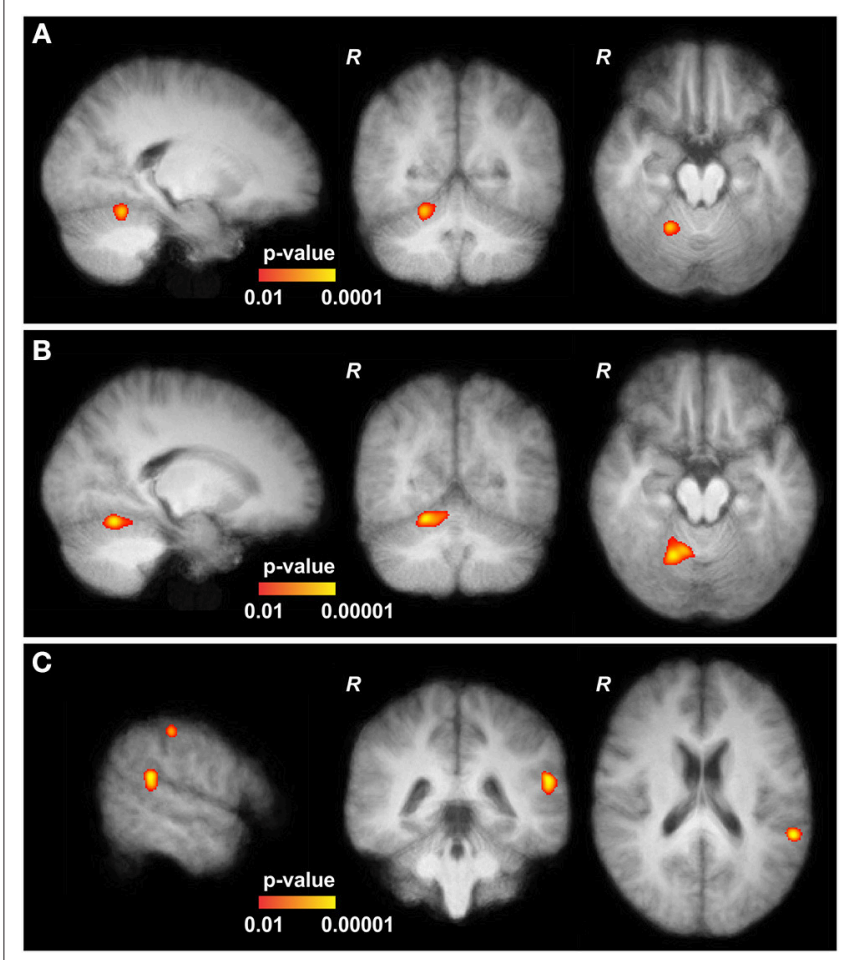

FIGURE 2 | Functional MRI data of cervical dystonia (CD) patients. (A) At baseline, the upper part of the cerebellum (CRB) of CD patients showed slightly increased activation in comparison to controls (MNI152 21, -54, -18). (B) After continuous theta-burst stimulation (CTBS), main and significantly increased activations in CD patients are shown in the upper part of the right CRB (MNI152 19, -59, -16, adjacent to the baseline results, further pronounced). (C) Two other significantly elevated activations were found adjacent to the gyrus angularis (MNI152 -57, -42, 21) and the postcentral sulcus (MNI152 -55, -27, 48). All depicted activations are overlayed on the average coregistered and linearly transformed brains of the subjects. Some moderate but significantly elevated activations in the left primary motor and primary somatosensory cortex and the left premotor cortex are not shown. Comparison with patients at baseline and after stimulation of the left dorsal premotor cortex (CRB vs. PMd) showed increased activations at the same locations and at the same significance levels with only minimal differences (not shown).

stimulation ( $143 \pm 30 \mathrm{~ms}$ vs. $160 \pm 34 \mathrm{~ms}$; adjusted $p=0.006)$, but not after CRB or sham stimulation (Figure 3B). cTBS did not have a significant effect on MEP amplitudes, neither in CD patients, nor in controls (Figure 3B).

\section{Clinical Assessment}

There were no significant changes of TWSTRS and Tsui scores following cTBS at PMd (TWSTRS $-1.4 \pm 2.0, p=0.146$; Tsui $-0.3 \pm 1.8, p=0.837$ ), at CRB (TWSTRS $-0.2 \pm 2.7, p=0.816$; Tsui $-0.4 \pm 1.1, p=0.746$ ), or sham stimulation (TWSTRS -0.8 $\pm 2.4, p=0.705$; Tsui $+0.2 \pm 0.9, p=0.898$ ). Similarly, CGI-I remained stable after cTBS at any site.

\section{DISCUSSION}

The present study assessed the role of CRB in CD. Employing a multimodal approach comprising functional MR imaging, 


\section{A baseline MEP}

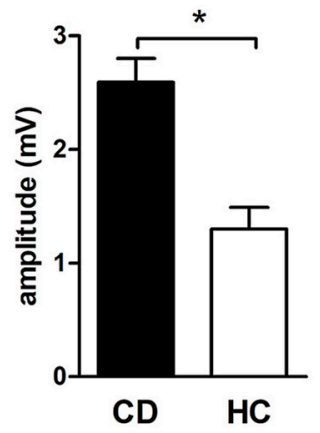

baseline CSP

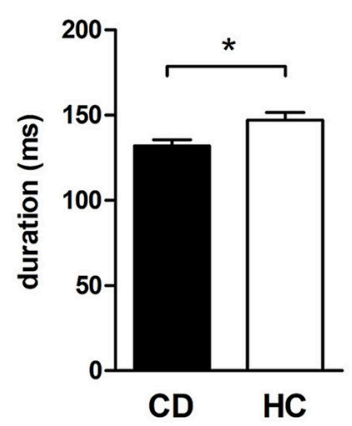

B

cTBS (CD)
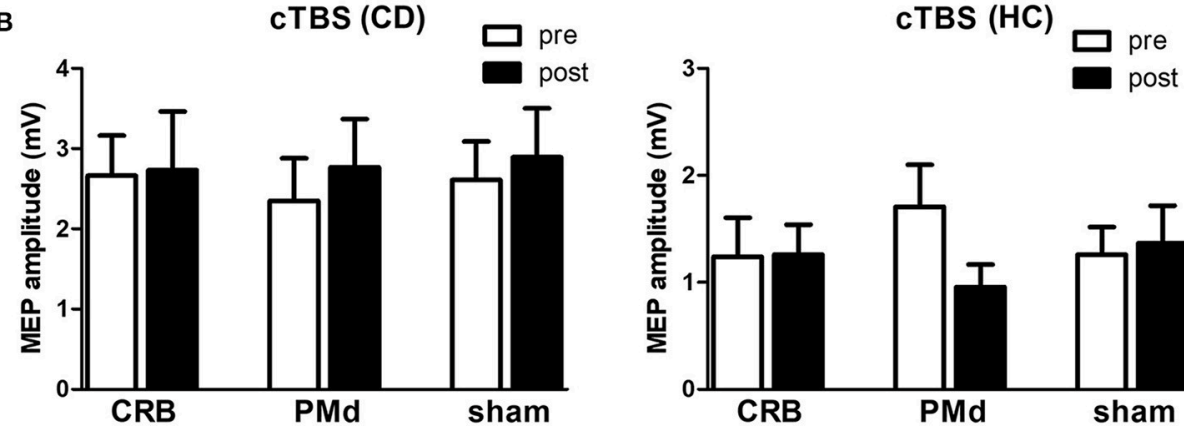

cTBS (HC)

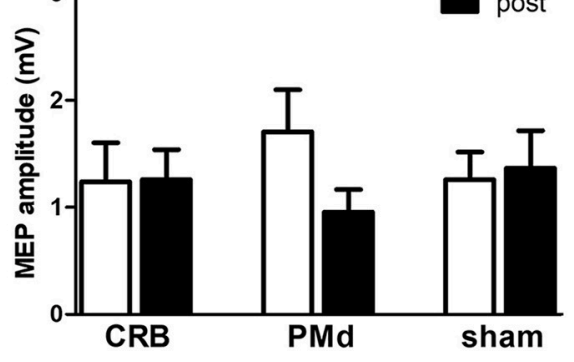

cTBS (CD)
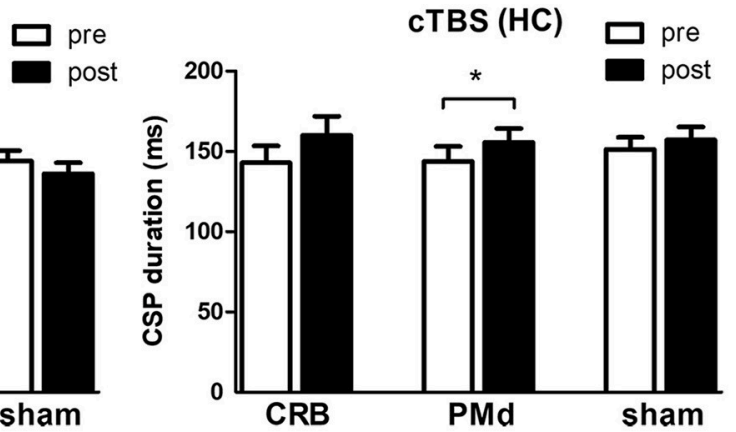

FIGURE 3 | Transcranial magnetic stimulation data. (A) Baseline mean motor-evoked potential (MEP) amplitudes and cortical silent period (CSP) duration in CD patients (CD) vs. healthy controls ( $\mathrm{HC}$ ). (B) Mean MEP amplitudes and CSP duration before and after cTBS at the cerebellum (CRB), dorsal premotor cortex (PMd), and sham in CD patients and healthy controls. ${ }^{*}$ indicates significant difference.

neurophysiological assessment, and blinded clinical rating, we found $\mathrm{CD}$ to be associated with increased brain activation during movement of the (clinically non-dystonic) right hand. Moreover, CD involves an impairment of cortical inhibitory mechanisms, as evidenced by a reduction of CSP duration. Cerebellar interference by TMS enhanced overactivation of CRB while it partially normalized cortical disinhibition. In the following, possible implications of our findings will be discussed.

\section{Finger-Tapping Related Brain Activation in CD}

Finger tapping of the right hand was associated with activation of contralateral M1 and ipsilateral CRB both in CD patients and controls. This is in line with a number of previous studies [e.g., (43-45)] and concurs well with common neuroanatomical knowledge. Combined anatomical, physiological, and imaging evidence suggests that voluntary movements are controlled by a network of regions, comprising motor cortex, basal ganglia, thalamus, dentate nucleus, and cerebellar cortex. CRB is commonly accepted to play a major role in motor task planning and coordination, integration of multisensory peripheral input, and feedback generation to the motor cortex.

At baseline, activation of the right cerebellar hemisphere was significantly increased in $\mathrm{CD}$ patients as compared to healthy controls. The elevated activation was located in the anterior lobe of the CRB. Projection of this area onto a map of the human CRB based on functional connectivity to cerebral networks (46) indicated that this part of the CRB is tightly connected to the hand motor area (Figure 4). Notably, increased cerebellar activation occurred during a simple motor task performed by a non-dystonic limb-a new finding as compared to previous studies in CD patients which did not report cerebellar 


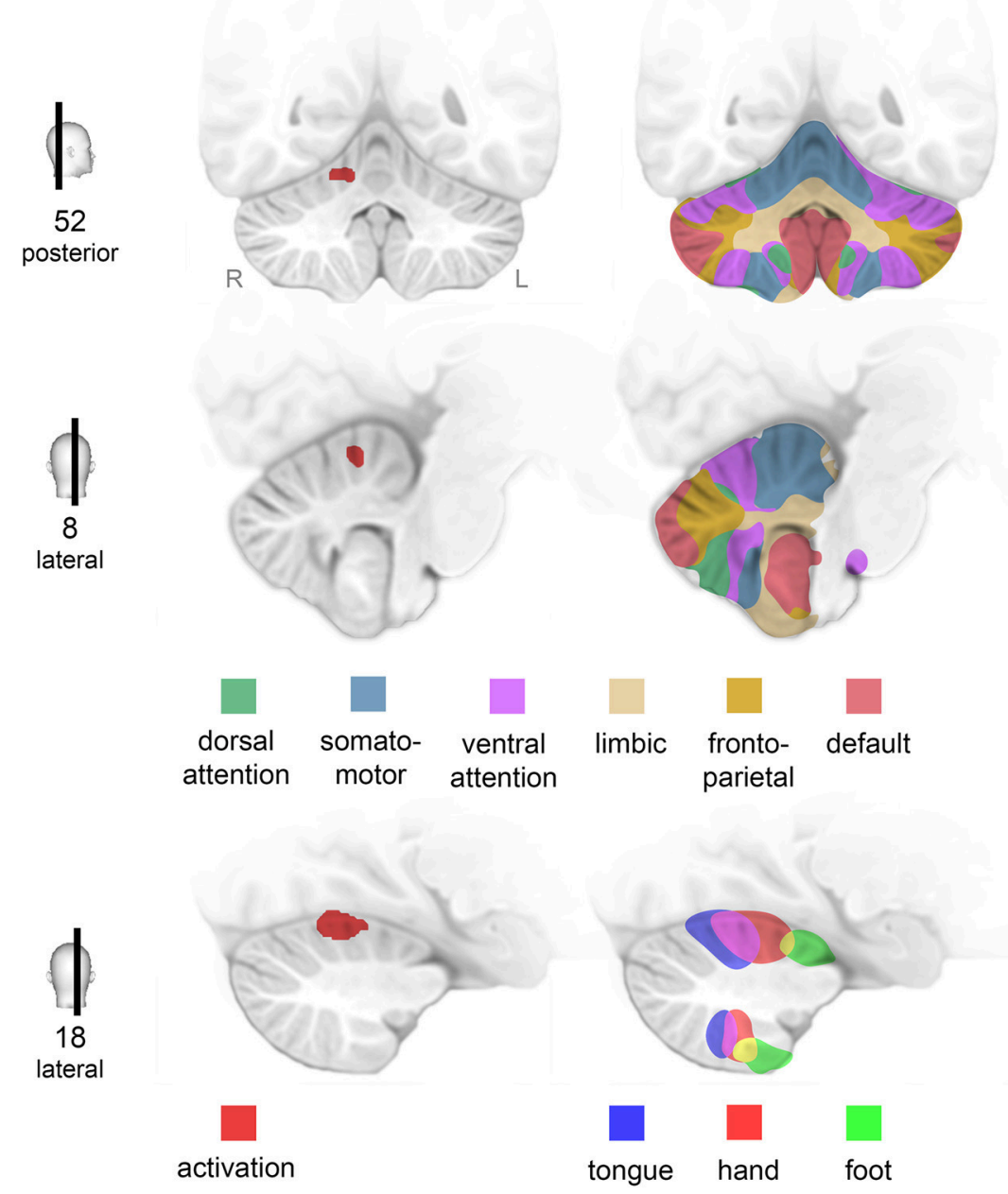

FIGURE 4 | Activation of specific networks in the cerebellum. Finger tapping of the right hand was associated with increased activation ( $p<0.0001)$ of the right lateral cerebellum (CRB) in patients with cervical dystonia compared to healthy controls (middle column). Projection on a map of the human CRB based on functional connectivity to seven major brain networks (46) reveals that this area of overactivation is strongly connected to the contralateral hand motor area (right column). Numbers (left column) indicate MNI-coordinates (posterior corresponds to y-coordinate, lateral to x-coordinate).

abnormalities during simple hand motor tasks $(19,20,47)$. Both the application of different motor tasks and the use of a scanner with higher magnetic field strength in our study (20) might contribute to this difference.

To interpret our finding, it seems crucial to discriminate reports on abnormalities derived from a clinically dystonic area from findings associated with a non-dystonic movement or even at rest. To our knowledge, only one functional imaging study assessed brain activation during head rotation in CD patients. While isometric (i.e., motionless) head rotation into the direction of the torticollis was associated with an increase of activation in the ipsilateral anterior CRB, isometric rotation into the opposite direction came along with increased activation in ipsilateral precentral and contralateral postcentral cortex regions (22). The authors propose a pathogenic role of the CRB, but compensatory role of the sensorimotor cortex in $\mathrm{CD}$, acknowledging that intentional muscle contraction might differ from involuntary head movements in CD (22).
In contrast, $\mathrm{CD}$ patients in the present study were asked to keep their head relaxed while performing a simple tapping task or resting. Within block design, any BOLD signal associated with task-free, CD-related or compensatory muscle contraction was dissolved by subtraction. Thus, cerebellar overactivation can be directly attributed to finger tapping. This may be interpreted as a result of motor overflow, i.e., an unintentional extension of tonic cervical activation into the representations of finger movements, which has become a core feature within the motor phenomenology of dystonic disorders $(1,48)$. Alternativelythough not mutually exclusively-cerebellar overactivation may be viewed as an indicator of a global "dystonic trait." Indeed, in a PET study, even completely asymptomatic DYT1 carriers showed increased cerebellar activity at rest (49). Similarly, nonmanifesting DYT1 mutation carriers performing at matched levels overactivated the lateral CRB and the right inferotemporal cortex during motor sequence learning compared to age-matched controls (50). Moreover, resting state fMRI revealed an increase 
of negative cerebello-cortical functional connectivity in patients suffering from writer's cramp who typically are asymptomatic during rest (51). Taken together, one might speculate that cerebellar overactivation during non-dystonic movements or rest may indicate an increased "demand" of tonic cerebellar activity to counter motor cortical overexcitability, well in line with a mainly compensatory role of the CRB $(5,16,52)$.

\section{Cortical Excitability}

At baseline, we found higher MEP amplitudes and decreased CSP duration in $\mathrm{CD}$ as compared to healthy controls, which is well in line with earlier studies providing evidence of motor cortical disinhibition and concurs with the overall pathophysiological concept of disturbed sensorimotor integration in CD.

Findings about MEP amplitudes in different forms of dystonia are inconsistent, with most studies describing normal (53-58) and only few describing higher $(53,57,59)$ amplitudes.

CSP is commonly accepted as a marker of cortical inhibitory capacity mediated by GABAergic transmission (60,61). Lower CSP duration has already consistently been described in patients suffering from writer's cramp (62), facial (63), and cervical dystonia $(57,64)$. In CD patients, a positive correlation of CSP duration recorded from the sternocleidomastoid muscle with symptom severity on the TSUI scale was reported, suggesting an impairment of inhibitory motor control to underlie the dystonic symptoms $(57,64)$. However, as CSP has been assessed remote from a clinically dystonic muscle in the majority of studies, reduced CSP duration may be viewed as another indicator of a global "dystonic trait" in CD patients.

In healthy controls, there was an increase of CSP duration after PMd stimulation. This is well in line with previous data showing reduced M1 excitability after applying this inhibitory protocol to PMd (65), possibly by depression of excitatory connections to M1. Conversely, the lack of an effect of PMd stimulation in the $\mathrm{CD}$ group might be interpreted as a further indicator of motor cortical disorganization in dystonia.

\section{Effects of Cerebellar cTBS on Finger-Tapping Related Brain Activation and CSP}

We applied cTBS to the lateral CRB in order to probe the effects of an excitability-modulating protocol on finger tapping related brain activation. We observed even pronounced additional activation of the ipsilateral CRB as well as significantly elevated activation of the contralateral sensorimotor region and the angular gyrus after cerebellar cTBS in CD patients-both compared to healthy controls and compared to baseline and PMd stimulation within the group of patients.

Suprathreshold TMS of the CRB has an inhibitory effect on contralateral M1 excitability, which is usually explained by activation of Purkinje cells leading to an inhibition of dentate nucleus and consequently less excitatory tonic output onto contralateral M1 via dentate-thalamo-cortical connections (34, 66-68). Notably, unilateral cerebellar cTBS, which is performed at subthreshold intensity, has also been shown to decrease contralateral MEP amplitudes (34,69-71). We therefore suggest that cTBS, rather than directly affecting the Purkinje cells, acts via transsynaptic modulatory effects on stellate and basket cells or parallel fibers within superficial layers of the CRB. As superficial layer cells are known to have inhibitory influence on Purkinje cells, cTBS-induced depression of these cells would eventually result in an inhibition of M1 excitability $(34,70,72)$.

It remains open whether activity dependent metaplastic effects, which have been shown to occur at M1 following muscle contractions prior to cTBS $(73,74)$, might also play a role at cerebellar stimulation. To this end, future studies in healthy subjects will need to disentangle the complex interplay of parameters with potential impact on the net effects of cerebellar cTBS, including motor activity and per interventional head position (58), respectively.

Following cTBS at CRB, we found a significant increase of CSP duration in CD patients. Given shortened CSP at baseline, this may indicate normalization of inhibitory mechanisms acting on M1 by a virtual lesion at the cerebellar hemispheres. The lack of an effect of cerebellar stimulation on CSP in the control group indicates differences between $\mathrm{CD}$ patients and controls in respect of their susceptibility to cerebellar "virtual lesions."

Application of cTBS to bilateral (as opposed to unilateral) CRB in our study confines direct comparison to a small number of previous studies $(26,70,75)$. Indeed, CSP did not change following unilateral cerebellar cTBS in CD patients (26), in PD patients (75), nor in healthy subjects $(26,70,75)$.

\section{Clinical Outcome}

We did not detect significant effects of cTBS on blindly-rated symptom severity of $\mathrm{CD}$, irrespective of the target site. A simple explanation might be that the impact of a single session of cTBS on the motor network is just too weak to provoke obvious clinical effects, e.g., due to network redundancy (76) and/or fast adaptive mechanisms (77). Our finding is here in line with comparable approaches using single session TBS (78). Notably, previous studies which reported clinical improvement of CD have applied at least 10 sessions of TBS $(26,27)$. Another reason might be a lack of sensitivity of our rating scales (TWSTRS, Tsui) for small clinical changes. Furthermore, it must be acknowledged that the aforesaid studies $(26,27)$ used the TWSTRS total score, while we exclusively collected the TWSTRS motor subscale. For instance, interventional effects on the pain subscale, as reported by Bradnam et al. (27), might have contributed significantly to changes of the TWSTRS total score. Finally, potential clinical effects were only assessed once, immediately after the intervention. Bearing in mind the delayed effects of deep brain stimulation in dystonia, it cannot be ruled out that protracted effects of cTBS have escaped our attention.

The fact that two protocols of TBS with opposite effects on the primary motor cortex, applied daily for 2 weeks, previously improved CD symptoms (26) might reveal that their global input on the network disorder itself is quite the same in spite of manifold local effects on cerebellar cortical structures (27). In view of the complex functional network of activating and inhibiting connections, parallel fiber and Purkinje cell interplay, and their dependency on climbing fiber activity, effects of interference by non-invasive stimulation of the cerebellar cortex 
is obviously hard to predict. A focal effect of TBS on one type of cerebellar neurons may therefore remain an over-simplified view.

\section{LIMITATIONS OF THE STUDY}

While our subgroups are rather small, they are comparable to previous physiological studies on $\operatorname{CD}(22,26,27)$, and group size has proved sufficient to show significant differences of brain activation and of physiological measures between groups. It cannot be ruled out, however, that small group sizes contributed to the lack of a significant TMS effect on the clinical scales of CD.

Another limitation might be a potential influence of previous neurotoxin treatment on our neurophysiogical and clinical data in spite of the fact that the experiments were performed at least 10 weeks after the last injection. While an even longer interval between drug application and experimental sessions would have been preferable, this has not been possible both for ethical reasons and for the sake of patient recruitment.

\section{CONCLUSION}

According to our multimodal approach, interpretation of fMRI data may benefit from physiological and/or clinical input. Given the lack of behavioral changes, the neurophysiological arm of the study may prove most useful to interpret the present findings: CSP, an established measure of cortical inhibitory capacity (79), was reduced in CD patients at baseline, but significantly increased toward normal duration following cerebellar stimulation. In other words, cerebellar cTBS may have partially restored the inhibitory net influence of the CRB on M1 within the cerebello-thalamo-cortical network. On fMRI, we found increased cerebellar activation during simple finger movements in CD patients compared to controls, which were even enhanced by cerebellar cTBS.

Altogether, we interpret our findings in favor of a compensatory role of the CRB within a network disorder underlying CD: If cerebellar overactivation during non-dystonic finger movements indicate a higher "demand" of tonic cerebellar activity to countervail overexcitability of the motor cortex, an

\section{REFERENCES}

1. Albanese A, Bhatia K, Bressman SB, Delong MR, Fahn S, Fung VS, et al. Phenomenology and classification of dystonia: a consensus update. Mov Disord. (2013) 28:863-73. doi: 10.1002/mds.25475

2. Lesser RP, Fahn S. Dystonia: a disorder often misdiagnosed as a conversion reaction. Am J Psychiatry. (1978) 135:349-52. doi: 10.1176/ajp.135.3.349

3. Berardelli A, Rothwell JC, Hallett M, Thompson PD, Manfredi M, Marsden CD. The pathophysiology of primary dystonia. Brain. (1998) 121( Pt. 7):1195212

4. Fahn S, Bressman SB, Marsden CD. Classification of dystonia. Adv Neurol. (1998) 78:1-10.

5. Shakkottai VG, Batla A, Bhatia K, Dauer WT, Dresel C, Niethammer M, et al. Current opinions and areas of consensus on the role of the cerebellum in dystonia. Cerebellum. (2017) 16:577-94. doi: 10.1007/s12311-016-0825-6

6. Prudente CN, Pardo CA, Xiao J, Hanfelt J, Hess EJ, Ledoux MS, et al. Neuropathology of cervical dystonia. Exp. Neurol. (2013) 241:95-104. doi: 10.1016/j.expneurol.2012.11.019 indirect, inhibitory net effect of cTBS on Purkinje cells may be able to enhance both cerebellar activation and M1 inhibition. This interpretation, though partly speculative, allows several predictions about measures of cortical excitability/inhibition and cerebello-cortical interactions which can be assessed systematically by future studies.

In conclusion, our combined approach of TMS and fMRI supports the hypothesis of general motor disorganization in CD, which remains subclinical in most body regions and therefore may be characterized a "dystonic endophenotype." Effects of non-invasive cerebellar interference point to a predominant compensatory function of cerebellar overactivation, which may act as a counterbalance of cortical disinhibition, a core feature of dystonic network disorders. Further research is needed to separate the specific contributions of the CRB in the control of dystonic vs. non-dystonic movements and to disentangle its complex interplay with basal ganglia circuits and the somatosensory system in the range of dystonic disorders.

\section{AUTHOR CONTRIBUTIONS}

DZ and JV designed the study protocol. DZ, TO, and GH planned the experiments. TO and GH carried out the study. DZ and JV advised on interpretation and analysis of the results. DZ, TO, and GH prepared the first draft of the manuscript. MR and JV reviewed the manuscript.

\section{FUNDING}

The study was supported by departmental research funds. The publication was funded by the German Research Foundation (DFG) and the University of Würzburg in the funding programme Open Access Publishing.

\section{ACKNOWLEDGMENTS}

Axel Haarmann assisted in the design of the figures. Thomas Musacchio supported recruitment of CD patients.
7. Teo JT, van de Warrenburg BP, Schneider SA, Rothwell JC, Bhatia KP. Neurophysiological evidence for cerebellar dysfunction in primary focal dystonia. J Neurol Neurosurg Psychiatry. (2009) 80:80-3. doi: 10.1136/jnnp.2008.144626

8. Batla A, Sanchez MC, Erro R, Ganos C, Stamelou M, Balint B, et al. The role of cerebellum in patients with late onset cervical/segmental dystonia?evidence from the clinic. Parkinson. Related Disord. (2015) 21:1317-22. doi: 10.1016/j.parkreldis.2015.09.013

9. Kadota H, Nakajima Y, Miyazaki M, Sekiguchi H, Kohno Y, Amako M, et al. An fMRI study of musicians with focal dystonia during tapping tasks. J Neurol. (2010) 257:1092-8. doi: 10.1007/s00415-010-5468-9

10. Filip P, Gallea C, Lehericy S, Bertasi E, Popa T, Marecek R, et al. Disruption in cerebellar and basal ganglia networks during a visuospatial task in cervical dystonia. Mov Disord. (2017) 32:757-68. doi: 10.1002/mds. 26930

11. Li Z, Prudente CN, Stilla R, Sathian K, Jinnah HA, Hu X. Alterations of resting-state fMRI measurements in individuals with cervical dystonia. Hum Brain Mapp. (2017) 38:4098-108. doi: 10.1002/hbm.23651 
12. Piccinin CC, Piovesana LG, Santos MC, Guimaraes RP, De Campos BM, Rezende TJ, et al. Diffuse decreased gray matter in patients with idiopathic craniocervical dystonia: a voxel-based morphometry study. Front Neurol. (2014) 5:283. doi: 10.3389/fneur.2014.00283

13. Egger K, Mueller J, Schocke M, Brenneis C, Rinnerthaler M, Seppi K, et al. Voxel based morphometry reveals specific gray matter changes in primary dystonia. Mov Disord. (2007) 22:1538-42. doi: 10.1002/mds.21619

14. Draganski B, Thun-Hohenstein C, Bogdahn U, Winkler J, May A. "Motor circuit" gray matter changes in idiopathic cervical dystonia. Neurology. (2003) 61:1228-31. doi: 10.1212/01.WNL.0000094240.93745.83

15. Vo A, Eidelberg D, Ulug AM. White matter changes in primary dystonia determined by 2D distribution analysis of diffusion tensor images. J Magnet Resonan Imag. (2013) 37:59-66. doi: 10.1002/jmri.23805

16. Argyelan M, Carbon M, Niethammer M, Ulug AM, Voss HU, Bressman SB, et al. Cerebellothalamocortical connectivity regulates penetrance in dystonia. J Neurosci. (2009) 29:9740-7. doi: 10.1523/JNEUROSCI.2300-09.2009

17. Delnooz CC, Pasman JW, Beckmann CF, van de Warrenburg BP. Taskfree functional MRI in cervical dystonia reveals multi-network changes that partially normalize with botulinum toxin. PLoS ONE. (2013) 8:e62877. doi: 10.1371/journal.pone.0062877

18. Magyar-Lehmann S, Antonini A, Roelcke U, Maguire RP, Missimer J, Meyer $\mathrm{M}$, et al. Cerebral glucose metabolism in patients with spasmodic torticollis. Mov Disord. (1997) 12:704-8. doi: 10.1002/mds.870120513

19. de Vries PM, Johnson KA, de Jong BM, Gieteling EW, Bohning DE, George MS, et al. Changed patterns of cerebral activation related to clinically normal hand movement in cervical dystonia. Clin Neurol Neurosurg. (2008) 110:1208. doi: 10.1016/j.clineuro.2007.09.020

20. Obermann M, Yaldizli O, de Greiff A, Konczak J, Lachenmayer ML, Tumczak F, et al. Increased basal-ganglia activation performing a nondystonia-related task in focal dystonia. Eur J Neurol. (2008) 15:831-8. doi: 10.1111/j.1468-1331.2008.02196.x

21. Burciu RG, Hess CW, Coombes SA, Ofori E, Shukla P, Chung JW, et al. Functional activity of the sensorimotor cortex and cerebellum relates to cervical dystonia symptoms. Hum Brain Mapp. (2017) 38:4563-73. doi: 10.1002/hbm.23684

22. Prudente CN, Stilla R, Singh S, Buetefisch C, Evatt M, Factor SA, et al. A functional magnetic resonance imaging study of head movements in cervical dystonia. Front. Neurol. (2016) 7:201. doi: 10.3389/fneur.2016.00201

23. Bohning DE, Shastri A, McConnell KA, Nahas Z, Lorberbaum JP, Roberts DR, et al. A combined TMS/fMRI study of intensity-dependent TMS over motor cortex. Biol. Psychiatry. (1999) 45:385-94

24. Walsh V, Rushworth M. A primer of magnetic stimulation as a tool for neuropsychology. Neuropsychologia. (1999) 37:125-35

25. Allam N, Brasil-Neto JP, Brandao P, Weiler F, Barros Filho J, Tomaz C. Relief of primary cervical dystonia symptoms by low frequency transcranial magnetic stimulation of the premotor cortex: case report. Arq de Neuro-Psiquiatr. (2007) 65:697-9. doi: 10.1590/S0004-282X2007000400030

26. Koch G, Porcacchia P, Ponzo V, Carrillo F, Caceres-Redondo MT, Brusa L, et al. Effects of two weeks of cerebellar theta burst stimulation in cervical dystonia patients. Brain Stimul. (2014) 7:564-72. doi: 10.1016/j.brs.2014. 05.002 .

27. Bradnam LV, McDonnell MN, Ridding MC. Cerebellar intermittent thetaburst stimulation and motor control training in individuals with cervical dystonia. Brain Sci. (2016) 6:56. doi: 10.3390/brainsci6040056

28. Huang YZ, Edwards MJ, Rounis E, Bhatia KP, Rothwell JC. Theta burst stimulation of the human motor cortex. Neuron. (2005) 45:201-6. doi: 10.1016/j.neuron.2004.12.033

29. Oldfield RC. The assessment and analysis of handedness: the Edinburgh inventory. Neuropsychologia. (1971) 9:97-113

30. Hoffland BS, Kassavetis P, Bologna M, Teo JT, Bhatia KP, Rothwell JC, et al. Cerebellum-dependent associative learning deficits in primary dystonia are normalized by rTMS and practice. Eur J Neurosci. (2013) 38:2166-71. doi: 10.1111/ejn.12186

31. Yousry TA, Schmid UD, Alkadhi H, Schmidt D, Peraud A, Buettner A, et al. Localization of the motor hand area to a knob on the precentral gyrus. A new landmark. Brain. (1997) 120 ( Pt 1):141-57.

32. Fink GR, Frackowiak RS, Pietrzyk U, Passingham RE. Multiple nonprimary motor areas in the human cortex. J Neurophysiol. (1997) 77:2164-74
33. Zeller D, Dang SY, Stefan K, Biller A, Bartsch A, Saur D, et al. Functional role of ipsilateral motor areas in multiple sclerosis. J Neurol Neurosurg Psychiatry. (2011) 82:578-83.doi: 10.1136/jnnp.2010.219964

34. Koch G, Mori F, Marconi B, Codeca C, Pecchioli C, Salerno S, et al. Changes in intracortical circuits of the human motor cortex following theta burst stimulation of the lateral cerebellum. Clin Neurophysiol. (2008) 119:2559-69. doi: 10.1016/j.clinph.2008.08.008

35. Del Olmo MF, Cheeran B, Koch G, Rothwell JC. Role of the cerebellum in externally paced rhythmic finger movements. J Neurophysiol. (2007) 98:14552. doi: 10.1152/jn.01088.2006

36. Theoret $\mathrm{H}$, Haque $\mathrm{J}$, Pascual-Leone A. Increased variability of paced finger tapping accuracy following repetitive magnetic stimulation of the cerebellum in humans. Neurosci Lett. (2001) 306(1-2):29-32. doi: 10.1016/S0304-3940(01)01860-2

37. Consky E, Basinski A, Belle L. The Toronto Western Spasmodic Torticollis Rating Scale (TWSTRS): assessment of validity and inter-rater reliability. Neurology. (1990) 40:445.

38. Tsui JK, Eisen A, Stoessl AJ, Calne S, Calne DB. Double-blind study of botulinum toxin in spasmodic torticollis. Lancet. (1986) 2:245-7

39. Busner J, Targum SD. The clinical global impressions scale: applying a research tool in clinical practice. Psychiatry. (2007) 4:28-37.

40. Jenkinson M, Bannister P, Brady M, Smith S. Improved optimization for the robust and accurate linear registration and motion correction of brain images. NeuroImage. (2002) 17:825-41. doi: 10.1006/nimg.2002.1132

41. Jenkinson M, Beckmann CF, Behrens TE, Woolrich MW, Smith SM. Fsl. Neuroimage. (2012) 62:782-90. doi: 10.1016/j.neuroimage.2011.09.015

42. Woolrich MW, Behrens TE, Beckmann CF, Jenkinson M, Smith SM. Multilevel linear modelling for FMRI group analysis using Bayesian inference. NeuroImage. (2004) 21:1732-47. doi: 10.1016/j.neuroimage.2003.12.023

43. Reichenbach JR, Feiwell R, Kuppusamy K, Bahn M, Haacke EM. Functional magnetic resonance imaging of the basal ganglia and cerebellum using a simple motor paradigm. Magn Reson Imaging. (1998) 16:281-7

44. Witt ST, Laird AR, Meyerand ME. Functional neuroimaging correlates of finger-tapping task variations: an ALE meta-analysis. Neuroimage. (2008) 42:343-56. doi: 10.1016/j.neuroimage.2008.04.025

45. Habas C, Axelrad H, Nguyen TH, Cabanis EA. Specific neocerebellar activation during out-of-phase bimanual movements. Neuroreport. (2004) 15:595-9

46. Buckner RL, Krienen FM, Castellanos A, Diaz JC, Yeo BT. The organization of the human cerebellum estimated by intrinsic functional connectivity. J. Neurophysiol. (2011) 106:2322-45. doi: 10.1152/jn.00339.2011

47. Opavsky R, Hlustik P, Otruba P, Kanovsky P. Sensorimotor network in cervical dystonia and the effect of botulinum toxin treatment: a functional MRI study. J. Neurol. Sci. (2011) 306:71-5. doi: 10.1016/j.jns.2011.03.040

48. Sitburana O, Wu LJ, Sheffield JK, Davidson A, Jankovic J. Motor overflow and mirror dystonia. Parkinsonism Relat Disord. (2009) 15:758-61. doi: 10.1016/j.parkreldis.2009.05.003

49. Carbon M, Su S, Dhawan V, Raymond D, Bressman S, Eidelberg D. Regional metabolism in primary torsion dystonia: effects of penetrance and genotype. Neurology. (2004) 62:1384-90. doi: 10.1212/01.WNL.0000120541.97467.FE

50. Carbon M, Ghilardi MF, Argyelan M, Dhawan V, Bressman SB, Eidelberg D. Increased cerebellar activation during sequence learning in DYT1 carriers: an equiperformance study. Brain. (2008) 131(Pt. 1):146-54. doi: 10.1093/brain/awm243

51. Dresel C, Li Y, Wilzeck V, Castrop F, Zimmer C, Haslinger B. Multiple changes of functional connectivity between sensorimotor areas in focal hand dystonia. J. Neurol Neurosurg. Psychiatry. (2014) 85:1245-52. doi: 10.1136/jnnp-2013-307127

52. Lehericy S, Tijssen MA, Vidailhet M, Kaji R, Meunier S. The anatomical basis of dystonia: current view using neuroimaging. Mov Disord. (2013) 28:944-57. doi: $10.1002 / \mathrm{mds} .25527$

53. Ikoma K, Samii A, Mercuri B, Wassermann EM, Hallett M. Abnormal cortical motor excitability in dystonia. Neurology. (1996) 46:1371-6.

54. Tinazzi M, Zarattini S, Valeriani M, Romito S, Farina S, Moretto G, et al. Long-lasting modulation of human motor cortex following prolonged transcutaneous electrical nerve stimulation (TENS) of forearm muscles: evidence of reciprocal inhibition and facilitation. Exp Brain Res. (2005) 161:457-64. doi: 10.1007/s00221-004-2091-y 
55. Abbruzzese G, Marchese R, Buccolieri A, Gasparetto B, Trompetto C. Abnormalities of sensorimotor integration in focal dystonia: a transcranial magnetic stimulation study. Brain. (2001) 124 (Pt. 3):537-45.

56. Zittel S, Helmich RC, Demiralay C, Munchau A, Baumer T. Normalization of sensorimotor integration by repetitive transcranial magnetic stimulation in cervical dystonia. J Neurol. (2015) 262:1883-9. doi: 10.1007/s00415-015-7789-1

57. Amadio S, Panizza M, Pisano F, Maderna L, Miscio C, Nilsson J, et al. Transcranial magnetic stimulation and silent period in spasmodic torticollis. Am J Phys Med Rehabilit. (2000) 79:361-8. doi: 10.1097/00002060-200007000-00007

58. Popa T, Hubsch C, James P, Richard A, Russo M, Pradeep S, et al. Abnormal cerebellar processing of the neck proprioceptive information drives dysfunctions in cervical dystonia. Sci Rep. (2018) 8:2263. doi: 10.1038/s41598-018-20510-1

59. Mavroudakis N, Caroyer JM, Brunko E, Zegers de Beyl D. Abnormal motor evoked responses to transcranial magnetic stimulation in focal dystonia. Neurology. (1995) 45:1671-7

60. Fuhr P, Cohen LG, Roth BJ, Hallett M. Latency of motor evoked potentials to focal transcranial stimulation varies as a function of scalp positions stimulated. Electroencephalogr Clin Neurophysiol. (1991) 81:81-9.

61. Chen R, Lozano AM, Ashby P. Mechanism of the silent period following transcranial magnetic stimulation. Evidence from epidural recordings. Exp Brain Res. (1999) 128:539-42.

62. Filipovic SR, Ljubisavljevic M, Svetel M, Milanovic S, Kacar A, Kostic VS. Impairment of cortical inhibition in writer's cramp as revealed by changes in electromyographic silent period after transcranial magnetic stimulation. Neurosci lett. (1997) 222:167-70

63. Curra A, Romaniello A, Berardelli A, Cruccu G, Manfredi M. Shortened cortical silent period in facial muscles of patients with cranial dystonia. Neurology. (2000) 54:130-5.

64. Pirio Richardson S. Enhanced dorsal premotor-motor inhibition in cervical dystonia. Clin Neurophysiol. (2015) 126:1387-91. doi: 10.1016/j.clinph.2014.10.140

65. Huang YZ, Rothwell JC, Lu CS, Wang J, Weng YH, Lai SC, et al. The effect of continuous theta burst stimulation over premotor cortex on circuits in primary motor cortex and spinal cord. Clin Neurophysiol. (2009) 120:796-801. doi: 10.1016/j.clinph.2009.01.003

66. Ugawa Y, Genba-Shimizu K, Rothwell JC, Iwata M, Kanazawa I. Suppression of motor cortical excitability by electrical stimulation over the cerebellum in ataxia. Ann Neurol. (1994) 36:90-6. doi: 10.1002/ana.410360117

67. Ugawa Y, Day BL, Rothwell JC, Thompson PD, Merton PA, Marsden CD. Modulation of motor cortical excitability by electrical stimulation over the cerebellum in man. J Physiol. (1991) 441:57-72.

68. Pinto $\mathrm{AD}$, Chen R. Suppression of the motor cortex by magnetic stimulation of the cerebellum. Exp Brain Res. (2001) 140:505-10. doi: $10.1007 /$ s002210100862

69. Li Voti P, Conte A, Rocchi L, Bologna M, Khan N, Leodori G, et al. Cerebellar continuous theta-burst stimulation affects motor learning of voluntary arm movements in humans. Eur J Neurosci. (2014) 39:124-31. doi: 10.1111/ejn. 12391

70. Harrington A, Hammond-Tooke GD. Theta burst stimulation of the cerebellum modifies the TMS-Evoked N100 potential, a marker of GABA inhibition. PLoS ONE. (2015) 10:e0141284. doi: 10.1371/journal.pone.0141284

71. Carrillo F, Palomar FJ, Conde V, Diaz-Corrales FJ, Porcacchia P, FernandezDel-Olmo M, et al. Study of cerebello-thalamocortical pathway by transcranial magnetic stimulation in Parkinson's disease. Brain Stimul. (2013) 6:582-9. doi: 10.1016/j.brs.2012.12.004

72. Evans GJ. Synaptic signalling in cerebellar plasticity. Biology Cell. (2007) 99:363-78. doi: 10.1042/BC20070010

73. Iezzi E, Conte A, Suppa A, Agostino R, Dinapoli L, Scontrini A, et al. Phasic voluntary movements reverse the after effects of subsequent theta-burst stimulation in humans. J Neurophysiol. (2008) 100:2070-6. doi: 10.1152/jn. 90521.2008

74. Gentner R, Wankerl K, Reinsberger C, Zeller D, Classen J. Depression of human corticospinal excitability induced by magnetic theta-burst stimulation: evidence of rapid polarity-reversing metaplasticity. Cereb Cortex. (2008) 18:2046-53. doi: 10.1093/cercor/bhm239

75. Kishore A, Popa T, Balachandran A, Chandran S, Pradeep S, Backer F, et al. Cerebellar sensory processing alterations impact motor cortical plasticity in Parkinson's disease: clues from dyskinetic patients. Cerebral cortex. (2014) 24:2055-67. doi: 10.1093/cercor/bht058

76. Friston KJ, Price CJ. Degeneracy and redundancy in cognitive anatomy. Trends Cogn Sci. (2003) 7:151-2. doi: 10.1016/S1364-6613(03) 00054-8

77. Wischnewski M, Schutter DJ. Efficacy and time course of theta burst stimulation in healthy humans. Brain Stimul. (2015) 8:685-92. doi: 10.1016/j.brs.2015.03.004

78. Bologna M, Paparella G, Fabbrini A, Leodori G, Rocchi L, Hallett M, et al. Effects of cerebellar theta-burst stimulation on arm and neck movement kinematics in patients with focal dystonia. Clin Neurophysiol. (2016) 127:3472-9. doi: 10.1016/j.clinph.2016.09.008

79. Uozumi T, Ito Y, Tsuji S, Murai Y. Inhibitory period following motor potentials evoked by magnetic cortical stimulation. Electroencephalogr Clin Neurophysiol. (1992) 85:273-9.

Conflict of Interest Statement: The authors declare that the research was conducted in the absence of any commercial or financial relationships that could be construed as a potential conflict of interest.

Copyright (C) 2019 Odorfer, Homola, Reich, Volkmann and Zeller. This is an openaccess article distributed under the terms of the Creative Commons Attribution License (CC BY). The use, distribution or reproduction in other forums is permitted, provided the original author(s) and the copyright owner(s) are credited and that the original publication in this journal is cited, in accordance with accepted academic practice. No use, distribution or reproduction is permitted which does not comply with these terms. 